\title{
OPTIMIZATION OF PERSONNEL COST IN AIRCREW ASSIGNMENT PROBLEM USING A SIMPLE FUZZY LOGIC APPROACH
}

\author{
Institut Teknologi Bandung \\ Department of Mathematics \\ Jl. Ganesha 10 Bandung, 40132 \\ Indonesia \\ novriana@math.itb.ac.id
}

Novriana Sumarti, Ferdyanto Chandra and Jeremy Minardi

\begin{abstract}
In aviation industries, the aircrew assignment problem is one of the most important factors in total operational cost optimization. This problem will be solved in two steps: flight pairing and aircrew scheduling. The constraints to be satisfied in flight pairing include having the same airport for first departure and final destination, and the limitations of flying time, duty time and transit time. The optimization process results in optimal flight pairings that minimize the number of personnel needed to serve a flight schedule over a given period of time. Further optimization is needed to obtain a schedule in which an aircrew team can serve a rotation with the largest possible number of pairings on the condition that all constraints are fulfilled. For aircrew scheduling, there are constraints on flying time, resting time, total number of takeoffs, and number of holidays and workdays. The investigated optimization process was designed to get optimal rotations along with maximum total personnel cost reduction. The data set used in this research is a onemonth full flight schedule from a big airline in Indonesia. A simple fuzzy logic approach was used to find a new flying time constraint in order to optimize personnel cost and evenly distribute the assignments. The results show that the new optimal flying time constraint can reduce personnel cost up to 5.07\% per month, so it can yield significant savings on a yearly basis.
\end{abstract}

Keywords:flight pairings, aircrew assignment problem, optimization, fuzzy logic, finance.

\section{Introduction}

The optimization problem dealing with allocation of optimal flight pairs and assignment of aircrews to each rotation is called the aircrew scheduling problem. Since the last decade of the 20th century many aspects of this problem have been discussed extensively, including computational techniques and the management of incidental problems. In [1], the authors consider the problem with a set of sectors (or flights) requiring 2 regular pilots and a supplementary crew (a third pilot) in some sectors to generate more cost-efficient scheduling. A heuristic procedure was used to solve the sequence of matching problems, i.e., a repeated matching algorithm.

In [2], the authors designed and implemented an aircrew assignment system based on the principle of artificial intelligence and the framework of abductive logic programming (ALP). Here, abduction is defined as the process of reasoning for explaining a given observation according to a general theory that describes the domain of observation. This approach gives a flexible modelling environment in which both the basic problem and its constraints can be easily represented directly from their high-level natural specification. Then an effective automatic translation (reduction) of this high-level representation is provided to the lower-level computational goals (constraints) that need to be solved. This approach is able to tackle the problem of adjusting or correcting existing solutions due to changes in the application environment.

In [3], the authors present a scatter search algorithm, which is a population-based meta-heuristic method in which solutions are intelligently combined to yield better solutions. Its aim is to assign a personalized roster to each aircrew member ensuring fairness among regular aircrew members considering each's preferences. This method has similar main steps as a genetic algorithm method. The result revealed efficient performance compared to variable neighbourhood search and branch-and-price methods.

In $[4,5$ and 6], the authors used a simulated annealing method, which is a technique based on an analogy between solving optimization problems and a simulation of the annealing of solids that minimize the energy of the states of the solid. This method is a search technique similar to the steepest descent algorithm, but it allows the occasional acceptance of an inferior solution in an effort to avoid being trapped in a local optimum. In [5 and 6], the authors applied their methods in solving a large-scale problem at a big airline company in Indonesia. The schedule was managed using the basic operations manual (BOM) containing general guidance for flight crew members regarding policies, procedures and aspects of flight operations that are applicable to all aircraft types.

In [7], the original approach of solving the problem of aircrew pairing consists of dividing it into a cyclic daily problem, a cyclic weekly problem, and a dated monthly problem. This approach prohibits the repetition of the same flight numbers in the pairing process. The authors proposed an alternative solution that exploits flight number repetition 
in pairings by skipping the first two phases and solving the monthly problem directly using a rolling horizon approach based on column generation.

A model applying various flexible strategies, in particular in the determination of shifts, squad members and working hours, has been proposed in [8] so that an airline can effectively manage its maintenance manpower supply. It uses a mixed integer program and implemented operating data from a leading Taiwan airline. In [9], the authors discuss the feasibility and cost efficiency of airline schedules in relation to incidental problems due to reactionary delays. They propose an indicator of stability to generate more robust aircraft and crew schedules. The integrated formulation of the problem results in a nonlinear stochastic recourse function. It is then decomposed into separate linear problems, which are solved using a heuristic iterative approach based on column generation and dynamic programming for the recourse functions.

In [10], the authors discuss the optimal length of prognostic distance, i.e., the time interval needed to gather information to predict future failures and to take appropriate action in order to decrease total cost related to a maintenance function. The model was implemented for minimizing life cycle costs and maximizing availability of aircraft across an airline network.

In [11], the authors propose to solve the aircrew recovery problem when a schedule is out of sync due to incidents of mechanical failure and bad weather. This method eliminates the step of rotation generation to allow the crew recovery problem to be dealt with directly in relation to flight legs. The model is about a hundred times faster than the traditional process. Data from an international airline in Taiwan were implemented in the model.

A fuzzy logic approach was used in [12] to solve the crew rostering problem, including the construction of personalized monthly schedules (rosters). Its multi-criteria decision making problem is solved using fuzzy control methods. For instance, fuzzy sets are used to describe the relative deviation of the assigned flight time from the ideal flight time or the assigned number of foreign per diem allowances from the ideal number. Defined fuzzy preferences are used, such as HN (highly negative relative deviation of the assigned value from the ideal), MN (moderately negative relative deviation of the assigned value from the ideal), $\mathrm{S}$ (small relative deviation of the assigned value from the ideal), MP (moderately positive relative deviation of the assigned value from the ideal), HP (highly positive relative deviation of the assigned value from the ideal) and ANY (any deviation whatsoever of the assigned value from the ideal). The approach was tested on data from airline companies characterized as small and medium sized.

In this paper we utilize a simple fuzzy logic approach for searching the optimal permitted flying time per pilot for a particular time period in order to optimize the total personnel cost. We will use the data and general guidance BOM from $[4,5]$. In the second section we explain the selection of flight pairings from given departure-arrival times in the schedule, and the determination of their binding conditions. In the third section, the objective functions and their constraints for solving the aircrew scheduling problem are formulated. A new set of flying times is proposed in the fourth section and the process of the fuzzy inference system to determine the new salary arrangement is explained. Finally, data implementation and discussion of the results are provided in the last 3 sections.

\section{Flight Pairing and Aircrew Scheduling Problems}

\subsection{Construction of Flight Couples and Pairings}

Restricted conditions determined by the airline become the constraints to be satisfied in constructing flight pairings. They are: having the same airport for the first departure and the final destination, and the limitations of flying time, duty time and transit time.

The airport of first departure and last arrival of a pairing should be the same. This airport is classified as the home base. In this case only 2 home base airports are considered out of 13 airports. There is also a regulation that any pair should contain one or more 2-flight sets that are taking off and landing from the same home base. For instance if A, B, $\mathrm{C}$ are the names of airports and $\mathrm{A}$ is the home base, the itinerary of pairing- $i$ containing only one 2 -flight set is $\mathrm{A}-\mathrm{B}-$ $\mathrm{A}$ and the itinerary of pairing $-j$ containing two 2-flight sets is $\mathrm{A}-\mathrm{B}-\mathrm{A}-\mathrm{C}-\mathrm{A}$. Due to this condition, the total number of flights in one time period of the schedule should be even.

Flying time is defined as the time period between the taking off and landing of the aircraft. Its maximum length for one pair is defined to be dependent on the number of pilots in the team: a regular 2-pilot crew team is allowed to have 9 hours flying time, an enlarged 3-pilot crew team can have 12 hours, and an enlarged 4-pilot crew team can have 22 hours. On the other hand, flight duty time (working hours) is defined as the time interval used by the aircrew team from the moment of report delivery upon departure at the beginning of a pair up to report delivery after the arrival at the end of a pair. The duration of each report delivery for one pair is 90 minutes. A one-day period is considered if the flying time is less than 14.5 hours and the duty time is less than 17 hours. A two-day period is considered if the flying time is between 14.5 and 22 hours and the duty time is between 17 and 34 hours.

Transit time is the time interval between the aircraft's landing from a previous flight and its next takeoff. The minimum transit time is 45 minutes or 0.75 hour. The actual length of transit time is an optimality consideration in constructing the flight pairs.

Before obtaining a pair consisting of two or more flights in one consecutive journey it is good to arrange all flights in couples, i.e., sets of 2-flights satisfying the constraints. Let $m$ be an (even) number of all flights in the schedule. The 
selection of the matched flights is conducted randomly. Let $\tilde{k}$ be the number of couples where $0<\tilde{k} \leq \frac{m}{2}$. Let $\tilde{x} \in$ $\mathcal{R}^{\tilde{k} \times m}$ be all possible flight couples and $\tilde{x}_{i}$ be the $i$-th couple for $i=1,2, \ldots, \tilde{k}$. There are 2 distinct indices $1 \leq i_{1}, i_{2} \leq$ $m$ so that $\tilde{x}_{i, i_{1}}=\tilde{x}_{i, i_{2}}=1$ and $\tilde{x}_{i, j}=0$ for $j \neq i_{1}, i_{2}$. All flights having been coupled, the next step is finding two or more couples that can be combined as a pair, using the technique introduced in [6].

Let $G \in \mathcal{R}^{\frac{m}{2} \times \frac{m}{2}}$ be the matrix of possible pairs, $G_{i j}=1$ if couple- $i$ and couple $-j$ can be paired for $j>i$, and 0 otherwise. Matrix $G$ is an upper triangular matrix. Let $D, E \in \mathcal{R}^{\frac{m}{2}}$ be the vectors containing the numbers of possible pairs with the next and previous couples respectively. Here $D$ is the result of the row-wise summation of matrix $G$, and $E$ is the result of the column-wise summation of matrix $G$.

$$
D_{i}=\sum_{j=i+1}^{m / 2} G_{i j}, E_{j}=\sum_{i=1}^{m / 2} G_{i j} .
$$

If $D_{p}=1$ and $E_{p}=0$ for $p=1,2, \ldots, \frac{m}{2}$, then couple $-p$ can be paired directly with couple $-p_{j}$ if $G_{p, p_{j}}=1$. In this case, the existence of index $p_{j}$ is unique. In the same way, $\operatorname{if} D_{p}=0$ and $E_{p}=1$, there is a uniquely existing $p_{i}$ such that $G_{p_{i}, p}=1$ so couple $-p$ can be paired directly with couple $-p_{i}$. For other nonzero values of $D_{i}$ and $E_{j}$, the construction of pairs will be done randomly. A pair can contain more than two couples on condition that all constraints are satisfied.

The construction of pairs yields matrix $x \in \mathcal{R}^{k \times m}$, where $k$ is the number of pairs. The values of $x_{i}, i=1,2, \ldots, m$ are 0 or 1 . For instance the first pair has $x_{1 i}=1$ for $i=1,3,4,8$, and $x_{1 j}=0$ for $j \neq i$. This means pair- 1 contains flight numbers 1, 3, 4 and 8 . There are no more possible flights that could be paired with those flights, considering the binding constraints. One pair of couples consists of matched flights that can be served by a team containing 2, 3 or 4 pilots. The value of $k$ also determines the number of aircrew members needed for one full flight schedule. We will choose the set of pairs $x$ that optimizes the number of possible pairs or the number of personnel needed to run one full schedule. This means it will find the minimum value of $k$. Let $F T(x) \in \mathcal{R}^{k}$ and $T T(x) \in \mathcal{R}^{k}$ be the total flying time and the transit time respectively resulted from pairing $x$.

$$
\min _{x} f(x, F T, T T) \text {. }
$$

Here $f: \mathcal{R}^{k \times m} \times \mathcal{R}^{k} \times \mathcal{R}^{k} \rightarrow \mathcal{R}$ is an objective function. One way to determine the objective function is defining the following function:

$$
H(x)=\sum_{i=1}^{k} F T(x)_{i}+\sum_{i=1}^{k} \frac{1}{45-T T(x)_{i}} .
$$

The first term of the function is the total length of the flying time in minutes and the second term is the summation of the inversion of the difference between the minimum transit time (45 minutes) and the real transit time. The first term's value is in hundreds and the second term's value is in negative tens, so they are appropriate values to be added up.

The pilot and his/her copilot(s) in a crew team could serve more than one pair during one period of the given schedule. The real required number of personnel can be reduced by finding the optimal scheduling, which is explained in the next section.

\subsection{Aircrew Scheduling}

There are four restricted conditions with respect to the time length of the schedule under observation, which in this case is one month. They are: the maximum length of flying time, the number of days off, the number of takeoffs, and the minimum length of rest period.

The maximum length of flying time depends on the license owned by the aircrew to fly a particular type of aircraft. In this case we use one type of aircraft to run the schedule and the maximum length of time considered here is 85 hours in one month. Later, this maximum length will become an open question in finding the optimal personnel cost for one month using fuzzy logic. In searching this new flying time constraint, there is an assumption that other regulations binding this maximum length are also satisfied.

There is an obligation for each aircrew member to take one day off after working 7 days in a row. The total number of days off in one month should be at least 8 . The maximum number of each aircrew member's takeoffs in one month is 90. The minimum length of rest period for each aircrew member is 9 hours.

In finding possible rotations for an aircrew team, the obtained pairs are combined in a consecutive way with the smallest length of total time every two pairs or more. The number of possibilities to combine is huge. However, based on experience, pairs with large flight numbers in their schedule have a smaller possibility than those with small flight numbers, which are usually the airports of popular destinations. Therefore, we begin with the large flight numbers and continue from there on.

Let $y \in \mathcal{R}^{n \times k}$ be a set of flight rotations, where $n$ is the number of rotations and $k$ is the minimum number of pairs obtained from the previous optimization result. For instance rotation-29 has $y_{29, j}=1$ for $j=1,4,5,6,11,15,29$, and $y_{29, i}=0$ for $i \neq j$. This means that the $29^{\text {th }}$-team of pilots serves pairings $x_{1}, x_{4}, x_{5}, x_{6}, x_{11}, x_{15}$ and $x_{29}$. We define $l(y) \in \mathcal{R}^{n}$ to be the number of crew members needed to serve the schedule for set of rotations $y$.

The objective of the aircrew scheduling task is to find the number of rotations that gives the minimum amount of total personnel cost. Let $F \operatorname{Tr}(y) \in \mathcal{R}^{n}$ and $S(y) \in \mathcal{R}^{n}$ be the flying time and the amount of total salary respectively for set of rotations $y$. Let $M S$ be a constant amount of basic salary per month if the pilot works with a flying time less than or equal to constant $A F T(y)$, i.e., the average flying time for one month. There will be an additional amount of salary if 
the crew member's flying time is more than $\operatorname{AFT}(y)$. The calculation for the additional amount is the multiplication between the extra hours and a constant amount of bonus money $S_{b}=0.75 \% M S$. The optimization problem for the minimum personnel cost needed for operating one full flight schedule is as follows:

$$
\min _{y} g(y, F T r, S)
$$

where $g: \mathcal{R}^{n \times k} \times \mathcal{R}^{n} \times \mathcal{R}^{n} \rightarrow \mathcal{R}$ is an objective function. One way of determining this objective function is

$$
Q_{1}(y)=\sum_{i=1}^{n} M S+\max \left\{F \operatorname{Tr}(y)_{i}-A F T(y)\right\} \cdot S_{b}
$$

which calculates the total salary for all aircrew members in set of rotations $y$.

We can set the objective function that can provide the arrangement of evenly distributed tasks among all crew members. The optimization problem for the minimum personnel cost needed for operating a one-month full flight schedule is as follows:

$$
\min _{y} g(y, F T r, A F T)
$$

The objective function can be in the following form:

$$
Q_{2}(y)=\frac{1}{\sum_{j=1}^{n} l(y)_{j}} \sum_{i=1}^{n} l(y)_{i}\left(M S+\max \left\{F \operatorname{Tr}(y)_{i}-A F T(y)\right\} \cdot S_{b}\right)
$$

We can see the difference between the results of these objective functions in the fifth section.

\section{A Simple Fuzzy Logic Approach for Optimizing the Flying Time Constraint}

We assume that the flying time constraint can still be modified so the optimal one can be found. The choice of this constraint is the only one that can significantly change the other constraints. A simple fuzzy logic approach is utilized for relaxing the limit of the permitted flying time per pilot for a particular period in order to solve the problem of optimizing the total personnel cost.

Let $\tilde{F}=\left[f_{L}, f_{M L}, f_{M R}, f_{R}\right]$ be a fuzzy number defining the permitted flying time. In the original optimization problem, the permitted flying time per crew member is $f_{M}=\frac{1}{2}\left(f_{M L}+f_{M R}\right)$ per month. The new optimal flying time constraint should be within $\widetilde{F}$, but intuively it is not far from $f_{M}$. It is straightforward that for $x \in \tilde{F}$ the minimum number of crew members needed decreases while the values of the average flying time per aircrew increase. Now we need to determine the basic salary, which depends on the average flying time for each value of the new flying time $\widetilde{F}$. We define 3 (three) types of salary for the aircrew, i.e., low, medium and high, which are represented by 3 (three) triangular fuzzy numbers, $\widetilde{L}, \widetilde{M}$ and $\widetilde{H}$, and their membership functions, $\mu_{\widetilde{L}}(x), \mu_{\widetilde{M}}(x)$, and $\mu_{\widetilde{H}}(x)$, respectively.

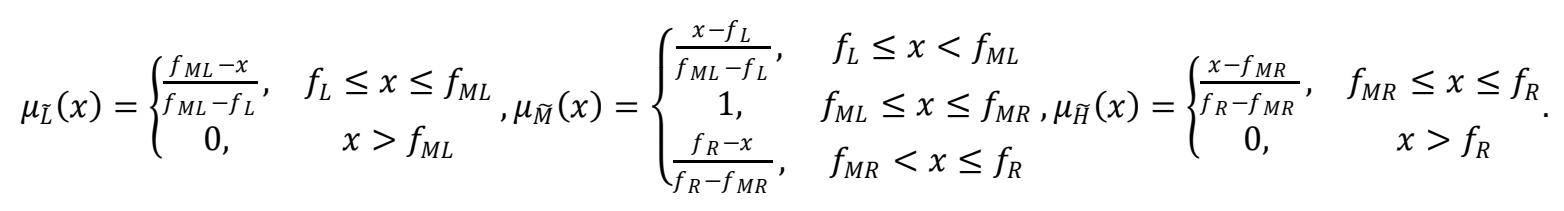

We need to define new basic salaries in order to make fair payment, because the lower flying time constraint will result in a smaller number of flights being able to be served by an aircrew team for the given period. On the other hand, the higher flying time constraint will result in a larger number of flights being able to be served by an aircrew team for the same period of time. Let $L S, M S$, and $H S$ be the new basic salaries, where $L S=(1-r) M S$ and $H S=(1+r) M S$, $0<r<1$. In the original problem, there is only $M S$ as the basic salary per hour.

Let $B S(x)$ be the basic salary per month for a new flying constraint for $x \in \widetilde{F}$. We define the basic salary based on the Sugeno method by the weighted average for $f_{L} \leq x \leq f_{H}$.

$$
B S(x)=\frac{\mu_{\tilde{L}}(x) \cdot L S+\mu_{\widetilde{M}}(x) \cdot M S+\mu_{\widetilde{H}}(x) \cdot H S}{\mu_{\widetilde{L}}(x)+\mu_{\widetilde{M}}(x)+\mu_{\widetilde{H}}(x)} .
$$

The amount of bonus money added on top of the basic salary is $S_{b}=0.75 \% B S$ per extra hour for each value of the average flying time. The optimum value of the total personnel cost is the minimum value of the multiplication between total flying time and salary. The total personnel cost depends on the determination of how far the values of $L S$ and $M S$ are from $M S$. A sensitivity analysis is conducted to see how the varying values of $r$ affect the optimal solution.

The process of aircrew scheduling from the second section is redone using each new flying time constraint. The objective functions used are Equations (3) and (4), which are the same as in the original problem.

\section{Numerical Results}

\subsection{Solving the Original Problem}

The data set used in solving the flight pairing problem here is a one-month full flight schedule from a big airline in Indonesia, which contains $m=702$ flights. There are 13 airports and 2 of them, CGK and DPS, become the home base airports. The optimization in pairing and scheduling processes follow the Monte-Carlo method developed using Matlab, where the next flight to be matched in the pairing process or the next pair to be united in the scheduling process is 
chosen randomly from the collection of possible candidates. The solution for the optimization problem is chosen as the best optimal value of the objective function. From the total of 702 flights, the pairing process results in 228 pairs that contain 2, 4 or 6 flights. The obtained total flying times are between 3.17 and 20.83 hours. The numbers of pairs containing 2, 3 and 4 pilots are 49, 43 and 136 pairs respectively. The total number of crew members needed to run the full schedule is 771 pilots. Examples of flight pairs can be found in Table 1.

The rotation-making process yields 40 rotations for the one-month flight schedule. Based on the average salary in Indonesia, the basic salary $M S=4000$ USD per month. We define bonus $S_{b}=300$ USD per extra hour. Table 2 shows an example of the results from the objective function in Equation (3), where the minimum of the total salary of all personnel is calculated. The flying time lengths vary from 29 to 85 hours, the flying time average is 76.40 hours and the average working day is 7.58 days. Notice that this flying time average is still far from the maximum length, 85 hours. With the total number of crew members at 146 pilots, the personnel cost is 603,890 USD per month.

Table 1: Examples of obtained flight pairs

\begin{tabular}{|c|c|c|c|c|}
\hline $\begin{array}{l}\text { Pair } \\
\text { no. }\end{array}$ & $\begin{array}{l}\text { Flight } \\
\text { nos. }\end{array}$ & $\begin{array}{l}\text { Flying } \\
\text { time } \\
\text { (hours) }\end{array}$ & $\begin{array}{l}\text { Flying } \\
\text { duty time } \\
\text { (hours) }\end{array}$ & $\begin{array}{l}\text { Nb of } \\
\text { crew }\end{array}$ \\
\hline 1. & $1,3,4,8$ & 7.00 & 11.50 & 2 \\
\hline 2. & 2,6 & 9.42 & 11.92 & 3 \\
\hline 3. & $5,11,17,24$ & 17.33 & 26.33 & 4 \\
\hline 4. & 7,10 & 3.17 & 5.67 & 2 \\
\hline 5. & 9,12 & 3.50 & 6.00 & 2 \\
\hline 6. & 13,19 & 4.58 & 12.67 & 2 \\
\hline 7. & 14,25 & 20.17 & 23.83 & 4 \\
\hline 17. & $36,51,56,57,62,67$ & 10.50 & 31.08 & 3 \\
\hline
\end{tabular}

Table 2: Examples of rotations obtained with objective function (3)

\begin{tabular}{llccc}
\hline $\begin{array}{l}\text { Rotation } \\
\text { no. }\end{array}$ & Pair nos. & $\begin{array}{l}\text { Nb of } \\
\text { flights }\end{array}$ & $\begin{array}{l}\text { Flying } \\
\text { time } \\
\text { (hours) }\end{array}$ & $\begin{array}{l}\text { Nb } \\
\text { of } \\
\text { crew }\end{array}$ \\
\hline 1. & 7,20 & 6 & 39 & 4 \\
2. & 10,23 & 8 & 35 & 4 \\
3. & $3,12,24,31,39$ & 20 & 84 & 4 \\
4. & $9,14,28,37$ & 14 & 63 & 4 \\
5. & $25,32,40,56$ & 20 & 75 & 4 \\
6. & $18,26,33,44$ & 12 & 76 & 4 \\
29. & $1,4,5,6,11,15,19$ & 20 & 36 & 2 \\
40. & $187,192,195,205$, & 16 & 82 & 3 \\
& $209,213,223,227$ & & & \\
\hline
\end{tabular}

Now we use the second objective function in Equation (4). Examples of the obtained rotations can be found in Table 3. The flying time lengths, the average flying times and the numbers of crew members needed are similar with the previous results. However, the total personnel cost is 603,350 USD per month.

Table 3: Examples of rotations obtained with objective function (4)

\begin{tabular}{llccc}
\hline $\begin{array}{l}\text { Rotation } \\
\text { no. }\end{array}$ & Pair nos. & $\begin{array}{l}\text { Number } \\
\text { of } \\
\text { flights }\end{array}$ & $\begin{array}{l}\text { Flying } \\
\text { time } \\
\text { (hours) }\end{array}$ & $\begin{array}{l}\text { Nb } \\
\text { of } \\
\text { crew }\end{array}$ \\
\hline 1. & 3,20 & 8 & 36 & 4 \\
2. & $10,23,39$ & 12 & 51 & 4 \\
3. & $7,12,28,37$ & 14 & 71 & 4 \\
4. & $14,24,31,40$ & 16 & 68 & 4 \\
5. & $18,26,33,42$ & 10 & 78 & 4 \\
6. & $25,32,44,56$ & 20 & 76 & 4 \\
25. & $2,8,13,19,19,22,35,38,11$ & 20 & 83 & 3 \\
40. & $143,167,171,178,180,185$, & 48 & 85 & 2 \\
& $186,189,196,207,211,212$, & & & \\
\hline
\end{tabular}




\subsection{Optimal Flying Time Problem}

Now, a simple fuzzy logic approach is used to find the new optimal flying time constraint. Set $\tilde{F}=[65,80,90,105]$, where the original problem has the flying time constraint $f_{M}=85$ hours per month. Figure 1 shows a decreasing trend in the number of crew members needed in order to serve the full schedule, declining from 194 to 121 . Due to the constant total amount of flying time for the full schedule, i.e., 11,154.76 hours, the ideal flying time per crew $F T_{I d}$ increases from 57.50 to 92.19 hours.

Letting $\widetilde{L}, \widetilde{M}$ and $\widetilde{H}$ be fuzzy numbers, the graphs of their membership functions are shown in Figure 2 . These fuzzy numbers and their membership functions are used to define the basic salaries if the flying time constraints vary from 65 to 105 hours. Here, the basic salary for the new flying time constraints varying from 80 hours to 90 hours are defined as the same as the basic salary for the previous flying time constraint at 85 hours.

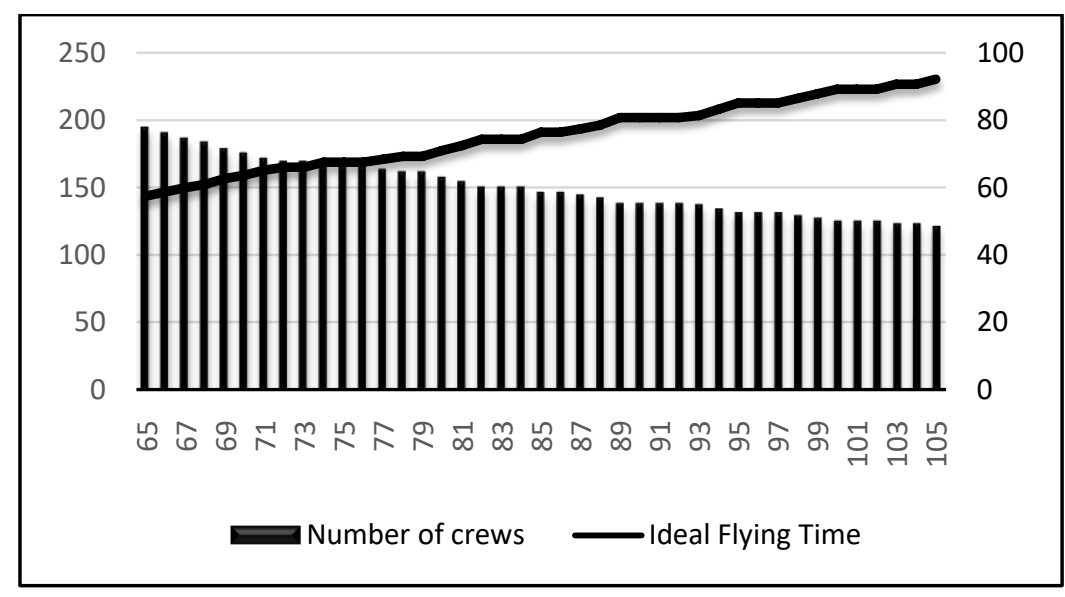

Figure 1: Number of crew members and the amount of ideal flying time.

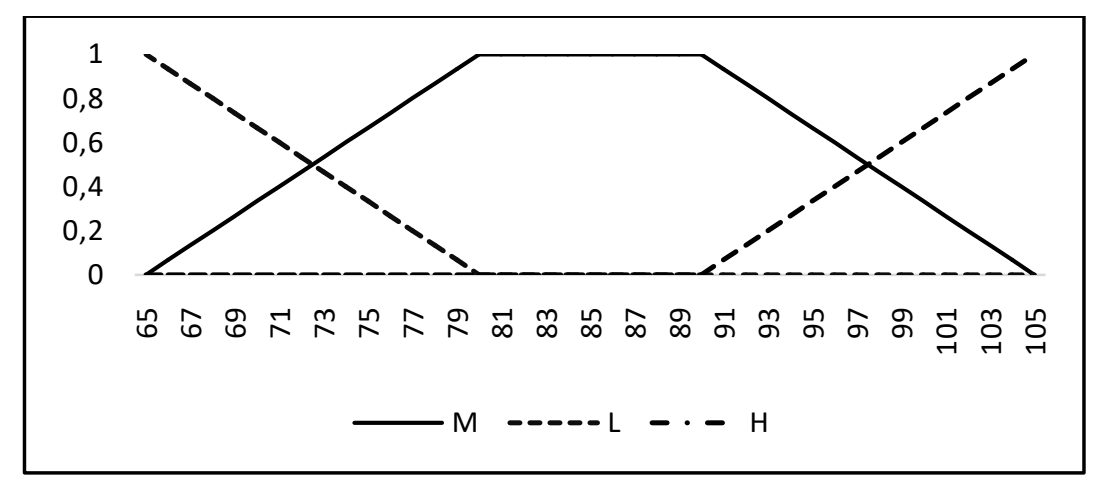

Figure 2: Fuzzy numbers $\widetilde{L}, \widetilde{M}$ and $\widetilde{H}$.

In the previous section, the basic salary per hour is $M S=4000$ USD. For $r=20 \%$, we define: low rate (LR) = 3200 USD, medium rate $(\mathrm{MR})=4000 \mathrm{USD}$, and high rate $(\mathrm{HR})=4800 \mathrm{USD}$. Using the weighted average, the basic salary for $65 \leq x \leq 105$, shown as a striped line in Figure 3,ranges from 3200 to 4800 USD. It increases in the interval from 65 to 80, remains constant at 4000 USD in the interval from 80 to 90 , and increases again in the interval from 90 to 105 . This new arrangement of the basic salary can be modified with respect to different values of $r$.

Using the right vertical axis, the total salary amount obtained by solving the optimality problem for each integer $x$ in $[65,105]$ can be seen in Figure 3. These amounts fluctuate above 600,000 USD when $x \leq 86$, below 600,000 USD when $87 \leq x \leq 101$, and increase when $x \geq 102$. The percentage changes of these total salary amounts with respect to $x=85$ can be found in Figure 4. The minimum amount of total salary in Figure 3 is 571,260 USD at $x=89$ hours. This is a $5.40 \%$ reduction from the amount obtained from the original problem at $x=85$, shown as the lowest point in Figure 4.

The choice of reduction percentage $r=20 \%$ is in fact the best one. Based on Figure 5, other values of $r$ give the lowest cost at the lowest flying time constraint $\mathrm{x}=65$. This flying time is too far from the original problem. Similar results are obtained when we use the objective function in Equation (4). If $x=85$, the average amount of salary per month per crew is 413,253.42 and the total amount of personnel cost is 603,350 USD. If $x=90$, for $r=0.2$ or $r=0.4$, the salary average is $403,352.11$, or a $2.39 \%$ decrease, and the personnel cost savings are $5.07 \%$. The decrease rate of personnel cost is larger than the decrease rate of average salary. If we compare to $x=72$ for 
$r=0.6$, the average salary is $281,544.44$, or a $31.87 \%$ decrease, and the personnel cost savings are $21.14 \%$. The decrease rate of personnel cost is smaller than the decrease rate of average salary. It follows that the most significant difference is produced by $r=0.2$.

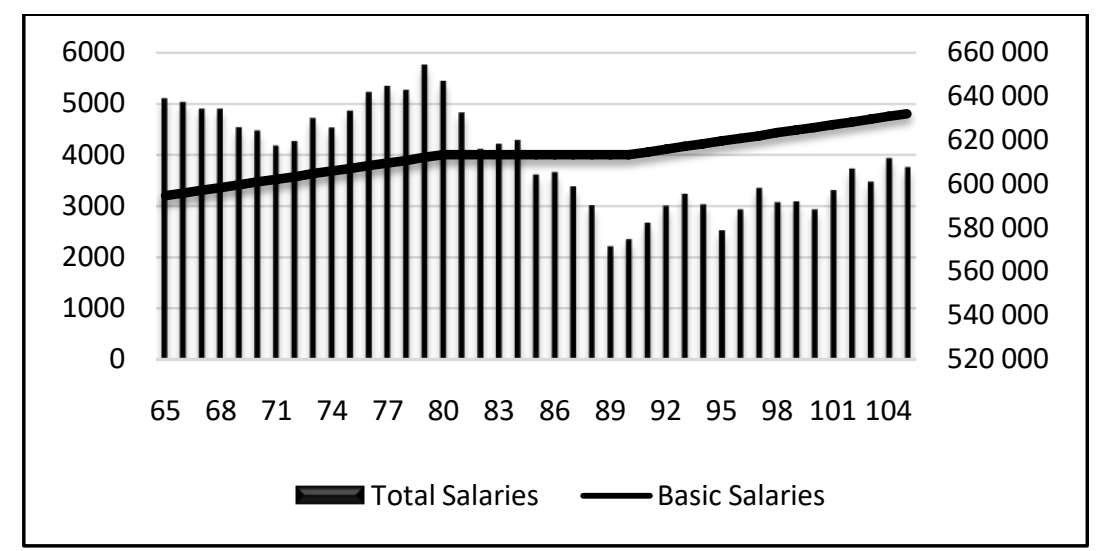

Figure 3: Basic salaries and total salaries.

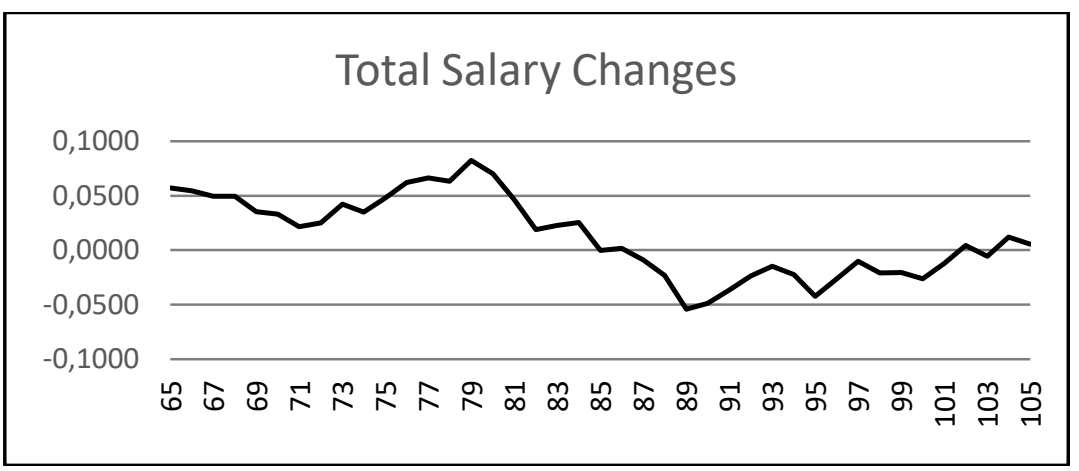

Figure 4: Total salary amount changes with respect to $x=85$.

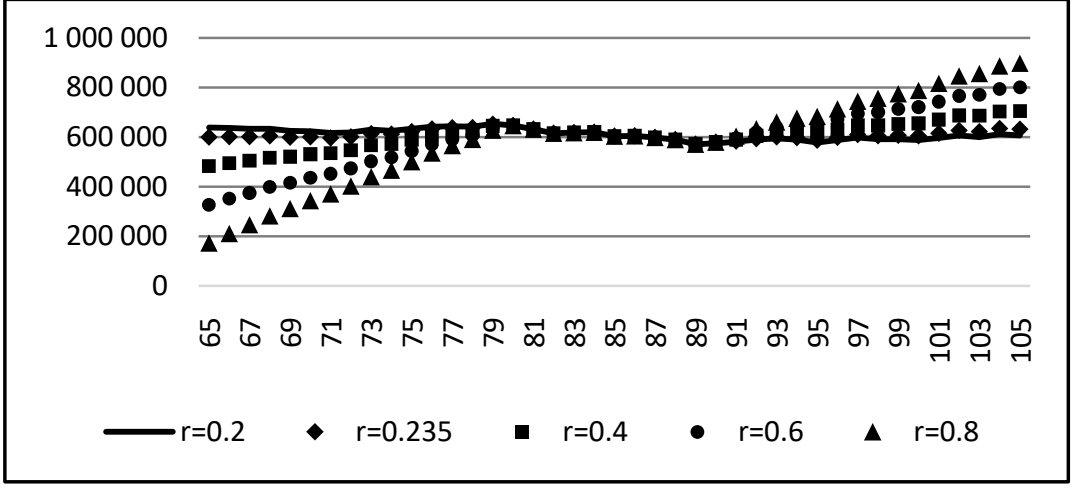

Figure 5: Sensitivity analysis on reduction percentage $r$.

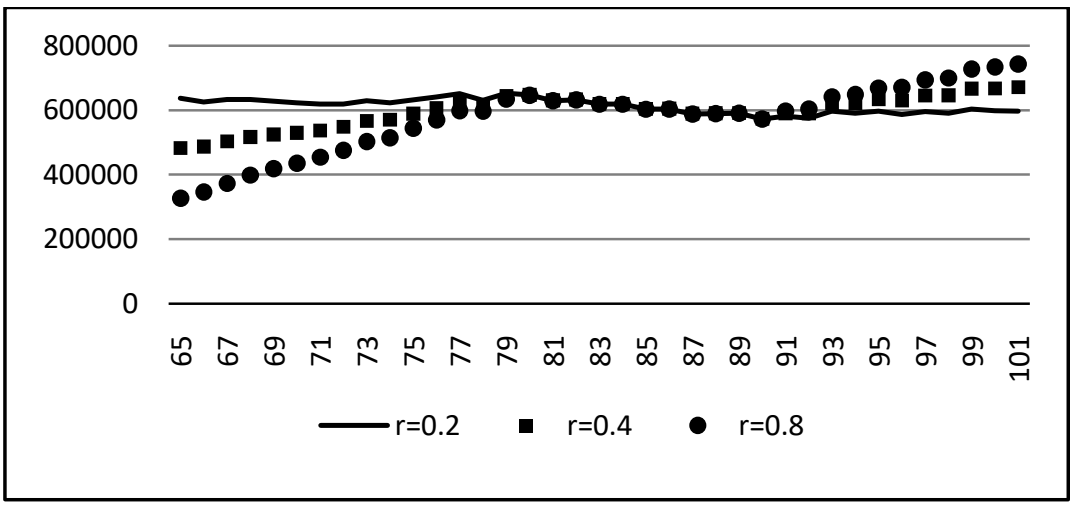

Figure 6: Sensitivity analysis on reduction percentage $r$ for evenly distributed salary. 


\section{Conclusions}

Based on data from 702 flights in a one-month schedule from a big airline in Indonesia using the original flying time limitation, the total number of flight pairings is 228 pairings, with 144 pairings for home base CGK and 84 pairings for home base DPS. The total number of crew members needed to operate the full schedule in one month is 146 pilots. The total personnel cost for one month is $603,890 \mathrm{USD}$, the average number of flights per pilot is 17.55 , and the average flying time per crew member is 76.40 hours. The average flying time is much lower than the flying time constraint per crew member of 85 hours per month.

Using a fuzzy logic approach and optimization of the amount of personnel cost, the new flying time limitation is 89 hours, so 138 pilots are needed; the average flying time per crew member is 80.83 hours and the total personnel cost is 571,260 USD. This means that a change in the flying time constraint from 85 to 89 hours can reduce total personnel cost up to $5.40 \%$. This reduction is very significant for the operation over a longer time period.

Having the optimization for an evenly distributed flying time, the new flying time limitation is 90 hours with 142 pilots and the average flying time per crew member is 78.55 hours. A change of the flying time constraint from 85 to 90 hours can reduce total personnel cost up to $5.07 \%$.

We conclude that a change in the flying time limitation reduces the optimal number of required crew members and the optimal personnel cost significantly. However, it is important to consider the impact of this longer flying time on the health and fitness of the crew members in executing their duties.In this case, the obtained result does not violate the civil aviation regulations [13,14] where the threshold is about $100-133$ hours per month. The model can be modified in terms of the size of the schedule, the applied regulations in determining the constraints, and theamount of basic salary in order to see the behaviour of the obtained solutions.

Acknowledgement: We are grateful to get a partial support from 2017 PM3I KK MIK ITB fund. We also thank to Dr. A.Y. Gunawan who gives significant input in this research.

\section{References}

[1] Wark, P., Holt, J., Ronnqvist. M., and Ryan, D. Aircrew schedule generation using repeated matching, European Journal of Operational Research 102. Pages 21-35 (1997).

[2] Kakas, A.C. and Michael, A. Air-Crew Scheduling through Abduction, Proceedings of IEA/AIE-99 (1999).

[3] Maenhout, B., and Vanhoucke, M. A Hybrid Scatter Search Heuristic for Personalized Crew Rostering in the Airline Industry, European Journal of Operational Research, 206 (1). Pages 155-167 (2010).

[4] Lucic, P. and Teodorovic, D. Simulated annealing for the multi-objective aircrew rostering problem, Transportation Research Part A 33. Pages. 19-45 (1999).

[5] Sumarti, N., Rakhman, R.N., Hadianti, R. and Uttunggadewa, S. Application of Simulated Annealing Method on Aircrew Assignment Problems in Garuda Indonesia, Proceeding of the 2012 International Conference of Applied and Engineering Mathematics (ICAEM'12), London, 4-6 July 2012.

[6] Hadianti, R., Novianingsih, K., Uttunggadewa, S., Sidarto, K.A., Sumarti, N., and Soewono, E. Optimization model for an airline crew rostering problem: Case of Garuda Indonesia, Journal of Mathematical and Fundamental Sciences, Vol. 45 (3). Pages 218-234 (2014).

[7] Saddoune, M., Desaulniers, G., and Soumis, F. Aircrew pairings with possible repetitions of the same flight number. Computers \& Operations Research 40. Pages 805-814 (2013).

[8] Yang, T., Yan, S., and Chen, H. An airline maintenance manpower planning model with flexible strategies. Journal of Air Transport Management 9. Pages 233-239(2003).

[9] Dück, V., Ionescu, L., Kliewer, N., and Suhl, L. Increasing stability of crew and aircraft schedules. Transportation Research Part C 20. Pages 47-61 (2012).

[10] Fritzsche, R., Gupta, J.N.D. and Lasch, R. Optimal prognostic distance to minimize total maintenance cost: The case of the airline industry. International Journal of Production Economics 151. Pages 76-88 (2014).

[11] Chang, S. A duty based approach in solving the aircrew recovery problem. Journal of Air Transport Management 19. Pages 16-20 (2012).

[12]Lucic, P. and Teodorovic, D. A fuzzy set theory approach to the aircrew rostering problem, Fuzzy Sets and Systems 95. Pages 261-271 (1998).

[13]Federal Aviation Administration (FAA), USA, https://www.faa.gov/regulations_policies/faa_regulations/ retrieved in June 2017.

[14]2011Civil Aviation Regulations, The Minister of Transport, ZA, http://www.sapfa.co.za/sites/default/files/CIVIL_AVIATION_REGULATIONS-2011.pdf, retrieved in June 2017. 